\title{
VCAM-1 Mediates Cigarette Smoke Extract Enhancement of Monocyte Adhesion to Human Carotid Endothelial Cells
}

\section{Authors \\ Gregory B. Pott, Ph.D. ', Mark Tsurudome, B.S. ${ }^{1}$, Jamie Bui, B.S. ${ }^{1}$, Charles Banfield, B.S. ${ }^{1}$, Sami Hourieh, B.S. ${ }^{1}$, Harsh Pratap, M.S. ${ }^{1}$, and Marc L. Goalstone, Ph.D ${ }^{1,2, *}$

\author{
Affiliations \\ ${ }^{1}$ Department of Medicine, \\ Metabolism and Diabetes, \\ The University of \\ Colorado Medical School, \\ University of Colorado \\ Denver Anschutz Medical \\ Campus, Aurora, \\ Colorado. \\ ${ }^{2}$ Research Service, Eastern \\ Colorado Health Care \\ System, Denver VA \\ Medical Center, Denver, \\ Colorado.
} Division of Endocrinology,}

\section{*Corresponding Author Marc Lee Goalstone, Ph.D. Associate Professor of Medicine Eastern Colorado Health Care System 1055 Clermont Street Denver, Colorado 80220, USA}

Ph\# 303-399-8020 x 3610 eM: Marc.Goalstone@va.gov

\begin{abstract}
:
Atherosclerosis is a cardiovascular disease that begins with inflammation and ends in decreased lumen diameter, occlusion and rupture if not addressed appropriately. Markers of vascular inflammation are, but not limited to, increased expression of Cell Adhesion Molecules on the vascular endothelium, recruitment of serum monocytes to the endothelium, and increases in the presence of inflammatory cytokines. Many factors can contribute to vascular inflammation and atherosclerosis, including diabetes, obesity, smoking, and genetic factors. In this report, we examine how cigarette smoke extract (CSE) contributes to the onset of vascular inflammation by (1) increasing VCAM-1 and ICAM1 expression in endothelium in a time- and dose-dependent manner, (2) affecting cytokine expression in endothelial cells, (3) increasing monocyte adhesion to carotid endothelial cells and (4) increasing the number of monocytes that migrate across a monolayer of carotid endothelial cells.
\end{abstract}

Keywords: cigarette smoke extract, monocytes, carotid endothelial cells 


\section{Introduction / Background}

The integrity of the vascular wall is an indication of the health of the artery as well as the circulatory system in general $[1,2]$. Many serum factors impact the wellbeing of the endothelial cells that line the arterial walls, including cytokines, monocytes and endocrine hormones [3]. Physical, biochemical and exogenous factors can adversely affect the integrity of the walls of the arteries and thereby activate sequelae of events that are detrimental to the integrity of the endothelial cell [4].

Many factors can contribute to the destructive remodeling of the endothelium in atherosclerosis, arterial inflammation and the onset of atherosclerosis in cardiovascular disease (CVD) incluing hyperinsulinemia in diabetes, advanced glycation end-products (AGE), hyperlipidemia and hypertension $[5,6]$. In addition, external environmental contaminants that are captured by the cardiovascular system can also wreak havoc on the endothelium. Along with internal serum factors that affect the vasculature, the soluble constituents of cigarette smoke may also find their way into the cardiovascular system [7]. Although smoking has been linked to lung disease, the impurities of smoke have been found in serum [8]. Their deleterious effects on the cardiovascular system are now being investigated.

Cellular Adhesion Molecules (CAMs) such as Vascular Cellular Adhesion Molecule-1 (VCAM-1) and Intercellular Cellular Adhesion Molecule-1 (ICAM-1) interact with ligands on the surface of macrophages and mediate the adhesion of monocytes to the endothelial surface [9]. Following the adhesion of monocytes to the endothelial surface, monocytes migrate into the vessel intima and recruit other leukocytes. Ultimately leading to vascular plaque formation[10]. VCAM-1 has been shown to be critical in the development of atherosclerotic lesions [9], and serum factors such as hyperinsulinemia, pro-inflammatory cytokines such as tumor necrosis factor alpha (TNF $)$, and bacterial lipopolysaccharides (LPS) can selectively induce VCAM-1 expression [11].

In this report, we show that cigarette smoke extract (CSE) (1) substantially increases the expression of VCAM-1, but not ICAM-1, on the surface of Human Carotid Endothelial Cells (HCEC), (2) decreases cytokine production in HCEC, and (3) increases the numbers of monocytes that bind to HCEC and migrate across an HCEC monolayer.

\section{Methods}

\subsection{Materials}

Primary Human carotid endothelial cells (HCEC) were obtained from Cell Applications, Inc. (San Diego, CA) and cultured in MesoEndo Cell Growth Medium (MGM, Cell Applications, Inc.). U937 human monocytes were obtained from American Type Culture Collection (ATCC, Manassas, VA) and cultured in RPMI medium with L-glutamine (Gibco/Invitrogen, Carlsbad, CA) supplemented with $10 \%$ heat inactivated Fetal Bovine Serum (Gibco) and 1\% Antimycotic-Antibiotic solution (Gibco). Cigarette Smoke Extract (CSE) was obtained from Murty Pharmaceuticals, Inc. (Lexington, KY) and reconstituted in dimethyl sulfoxide (DMSO, Sigma-Aldrich, St. Louis, MO, USA) to a stock concentration of $10 \mathrm{mg} / \mathrm{mL}$. APCconjugated anti-human ICAM-1, PEconjugated anti-human VCAM-1, and FITC- 


\section{VCAM-1 Mediates Cigarette Smoke Extract Enhancement of Monocyte Adhesion to Human Carotid Endothelial Cells}

conjugated anti-human CD14 antibodies were obtained from BD Pharmingen (San Jose, CA). Recombinant human MCP-1 was obtained from PeproTech (Rocky Hill, $\mathrm{NJ})$. All general lab reagents were purchased from Sigma- Aldrich (St. Louis, $\mathrm{MO})$.

\subsection{Stimulation and Flow Cytometric Analyses of Cellular Adhesion Molecules}

HCEC were cultured in MGM at $37^{\circ} \mathrm{C}, 5 \% \mathrm{CO}_{2}$ atmosphere in 6-well tissue culture dishes (ThermoFisher Scientific, Waltham MA) until confluent. Prior to stimulation of HCEC by CSE, spent growth medium was removed, cells were washed twice with PBS and fresh growth medium was added to the cells. Cells were introduced to indicated concentrations of CSE for indicated times. After stimulation, cell-free supernatants were collected and stored at $-80^{\circ} \mathrm{C}$ until later analysis for cytokine quantification. The cells were washed twice with $2 \mathrm{~mL}$ of $1 \mathrm{X}$ PBS (Gibco), and after the final PBS wash was aspirated, $0.5 \mathrm{~mL}$ of Cell Dissociation Solution Non-Enzymatic (Sigma-Aldrich, St. Louis, MO, USA) was added to each well. After incubating the cells at $37^{\circ} \mathrm{C}$ and $5 \% \mathrm{CO}_{2}$ for $30 \mathrm{~min}, 1 \mathrm{~mL}$ of $1 \%$ Bovine Serum Albumin (BSA, Sigma-Aldrich) in PBS was added to the cells and the cells were gently triturated into single cell suspensions. The cells were transferred to 5 $\mathrm{mL}$ Falcon polystyrene round bottom tubes (ThermoFisher Scientific) and centrifuged at $500 \mathrm{x} \mathrm{g}$ for $5 \mathrm{~min}$. After aspirating the supernatants, the cells were resuspended in 3 $\mathrm{mL} 1 \%$ BSA, pelleted at $500 \mathrm{x} \mathrm{g}$ by centrifugation, and the supernatants were removed by aspiration. The cells were resuspended in $200 \mu \mathrm{L}$ of $1 \% \mathrm{BSA}$, and 5 $\mu \mathrm{L}$ of PE-conjugated anti-VCAM-1 antibody and $5 \mu \mathrm{L}$ of APC-conjugated antiICAM-1 were added to cells and incubated in the dark for $30 \mathrm{~min}$ at room temperature.
The cells were washed twice with $3 \mathrm{~mL} 1 \%$ BSA and resuspended in 200

$\mu \mathrm{L}$ of $1 \%$ paraformaldehyde (PFA, Electron Microscopy Sciences, Hatfield, PA). After fixing the cells in PFA for $5 \mathrm{~min}$ at room temperature, the cells were diluted with an additional $300 \mu \mathrm{L}$ of PBS and analyzed using flow cytometry. The experiments were run on a BD LSRII (BD Biosciences, San Jose, CA). Mean Fluorescence Intensity (MFI) and gating percentages as part of data analysis was done using BD FACSDiva v6 software.

\subsection{Monocyte Adhesion Assay}

HCEC were cultured in MGM at $37^{\circ} \mathrm{C}, 5 \% \mathrm{CO}_{2}$ in 6-well tissue culture dishes until confluent. After aspirating the spent medium, fresh medium containing CSE was added to the cells. After incubating for $24 \mathrm{hr}$, the medium was aspirated, the cells were washed twice with $2 \mathrm{~mL}$ warm PBS, and $1 \mathrm{~mL}$ of fresh MGM was added to each well. U937 cells were centrifuged and resuspended in fresh MGM, and $1 \times 10^{6}$ cells were added to the HCEC monolayers in a $0.5 \mathrm{~mL}$ volume. The cocultured cells were rocked slowly for $1 \mathrm{~h}$ at $37^{\circ} \mathrm{C}, 5 \% \mathrm{CO}_{2}$. The medium was aspirated and unbound U937 cells were removed using three washes of PBS. Co-bound U937 cells and HCEC were re-suspended using Cell Dissociation Solution Non-Enzymatic as described above. U937 monocytes were stained in the co-cultures with FITCconjugated anti-human CD14 antibody, and total numbers of unstained cells (HCEC) and FITC-stained U937 cells were quantified using flow cytometry.

\subsection{Monocyte Transmigration assay}

HCEC were cultured in MGM at $37^{\circ} \mathrm{C}, 5 \% \mathrm{CO}_{2}$ on $8 \mu \mathrm{m}$ pore size membrane inserts (ThermoFisher Scientific) in 6-well tissue culture dishes until confluent. After aspirating the spent medium, fresh medium 


\section{VCAM-1 Mediates Cigarette Smoke Extract Enhancement of Monocyte Adhesion to Human Carotid Endothelial Cells}

was added to the upper $(2.5 \mathrm{~mL})$ and lower (3.0 mL) chambers. Monocyte Chemotactic Protein (MCP)-1 was added to the lower chamber as a monocyte chemoattractant to a final concentration of $20 \mathrm{ng} / \mathrm{mL}$, and various concentrations of CSE were added to the upper chamber. U937 cells were centrifuged and re-suspended in MGM and $1 \times 10^{6}$ cells were then added to the upper chamber in a $0.1 \mathrm{~mL}$ volume. The cocultures were incubated at $37^{\circ} \mathrm{C}, 5 \% \mathrm{CO}_{2}$ for $48 \mathrm{hr}$. The insert was removed and the transmigrated U937 cells present in the lower chamber were centrifuged, washed twice with $1 \%$ BSA, and stained with FITCconjugated anti-human CD14 antibody as described above. The total number of FITCstained U937 cells were quantified using flow cytometry.

\subsection{Cytokine Analysis}

Cell-free supernatants were collected from time course and dose response assays of CSE-treated HCEC and stored at $-80^{\circ} \mathrm{C}$ until analysis for cytokine quantification. Human Granulocyte Macrophage ColonyStimulating Factor (GMCSF), Interleukin (IL)-6, IL-8, MCP-1, MCP-2, and Regulated on Activation, Normal $\mathrm{T}$ Cell Expressed and Secreted (RANTES) were measured using a multiplex array chemiluminescence ELISA assay (Quansys Biosciences, Logan, UT, USA).

\subsection{Data Analysis}

Data were analyzed by either unpaired Student's $t$ test (two groups) or ANOVA with subsequent Tukey posttest (several groups) as indicated. A "P" value of less than 0.05 was considered significant. Results were expressed as the mean \pm Standard Error of the Mean (SEM) of three or more independent experiments.

\section{RESULTS}

In time course experiments we measured changes in the levels of VCAM-1 and ICAM-1 on the surface of HCEC in the absence or presence of CSE. Confluent HCEC were challenged without or with 50 $\mu \mathrm{g} / \mathrm{mL}$ of CSE for up to $48 \mathrm{hr}$ (Fig 1). CSE stimulation not only caused increases in the percentage of cells expressing VCAM-1 (Fig 1A), but the density of VCAM-1 molecules present on the cell surface (as determined by MFI) also increased (Fig 1B). VCAM-1 expression appeared to be maximal at $24 \mathrm{hr}$ of stimulation. Although the number of HCEC that expressed surface ICAM-1 did not change appreciably over time (Fig 1C), the surface density of ICAM1 molecules significantly $(\mathrm{P}<0.005)$ peaked at $6 \mathrm{hr}$ (Fig 1D), but decreased at later time points to near control levels (zero time).

In subsequent experiments, we performed dose response assays in order to determine optimal concentrations of CSE that would elicit maximum CAM expression over a $24 \mathrm{hr}$ time period. Increasing concentrations of CSE resulted in a significant expansion of HCEC expressing VCAM-1 (Fig 2A) and escalation of surface VCAM-1 density (Fig 2B). In contrast, while there were significant increases in HCEC cells expressing ICAM-1 at 20-50 $\mu \mathrm{g} / \mathrm{mL}$ CSE (Fig 2C), the increases were relatively minor and any increases in the density of surface ICAM-1 were not significant (Fig 2D).

Serum cytokines and chemokines are soluble molecules secreted by affected cells that act in concert with, and recruit cellular constituents of, the circulatory system. We wanted to determine whether cytokine expression in HCEC was affected by CSE over a $48 \mathrm{hr}$ period. Using $50 \mu \mathrm{g} / \mathrm{mL}$ CSE and various time points we observed increased amounts of GMCSF, IL-6, IL-8, MCP-1, MCP-2, and RANTES (Fig 3, white bars). However, when comparing cytokine expression of CSE-exposed cells (white 


\section{VCAM-1 Mediates Cigarette Smoke Extract Enhancement of Monocyte Adhesion to Human Carotid Endothelial Cells}

bars) to the no-CSE controls (black bars) at each time point, it became clear that CSE actually had a suppressive effect on cytokine expression in HCEC.

To confirm that CSE had a suppressive effect on cytokine production in HCEC, we next performed CSE doseresponse experiments with HCEC. HCEC were challenged with $0-50 \mu \mathrm{g} / \mathrm{mL}$ of $\mathrm{CSE}$ for $24 \mathrm{hr}$ and soluble cytokines were determined by ELISA (Fig 4). The expression of GMCSF, IL-6, IL-8, MCP-1 and RANTES in CSE-exposed HCEC decreased compared to the CSE-negative cells, confirming that CSE has an immunosuppressive effect on HCEC. No MCP-2 could be detected in these experiments (data not shown).

Since the expression of surface VCAM-1increased due to CSE exposure (Fig 1), we investigated whether increasing numbers of monocytes would bind to HCEC in the presence of escalating concentrations of CSE. After incubating an HCEC monolayer with $0-50 \mu \mathrm{g} / \mathrm{mL}$ of CSE for 24hr, U937 monocytes were added to HCEC and co-cultured for $1 \mathrm{hr}$ to allow adherence. Increasing concentrations of CSE significantly augmented binding of monocytes to the HCEC monolayer (Fig 5).

Plaque formation in atherosclerosis is initially triggered by the migration of monocytes across the endothelial barrier into the intima of the arterial wall [12]. After finding enhanced adherence of monocytes to HCEC surfaces following CSE exposure (Fig 5), we continued our investigation of monocyte interaction with HCEC by measuring the numbers of monocytes that migrated across an HCEC monolayer in the absence and presence of various concentrations of CSE over 48hr. We observed a significant increase in the number of monocytes that transmigrated across the HCEC monolayer of cells in the presence of CSE at concentrations as low as $5 \mu \mathrm{g} / \mathrm{mL}$ (Fig 6, $\mathrm{P}<0.01$ ).

\section{Discussion}

Many biological factors, such as diabetes, obesity, and smoking, are associated with CVD [5] [13]. Early stages of CVD involve vascular inflammation [14], and left unchecked, vascular inflammation can lead to atherosclerosis, plaque formation and vessel rupture [15]. Associated with vascular inflammation is increased expression of CAMs on the surface of endothelial cells, rolling and adhesion of monocytes to the surface of endothelial cells, transmigration of monocytes into the intima of the vascular wall and the upregulation of inflammatory cytokines and chemokines $[16,17]$.

Initiation of plaque formation is intricately linked to the recruitment and adhesion of monocytes to vascular endothelial surfaces through interactions between endothelial CAMs and monocytic binding ligands. Because leukocyte recruitment to the endothelium is dependent on CAM expression, there has been much attention focused on the impact of smoking on CAM expression by vascular endothelial cells. Schaberg et al [18] found that ICAM1(but not VCAM-1) expression was increased in lung peripheral pulmonary vessels in smokers compared to nonsmokers. Adams et al [19] found similar results with increased ICAM-1, but not VCAM-1, expression in Human Umbilical Vein Endothelial Cells (HUVECs) exposed to smoker's serum. In contrast, another study demonstrated that both ICAM-1 and VCAM-1 increased in HUVECs exposed to cigarette smoke condensate [20]. In animal studies where rats were exposed to cigarette smoke, higher levels of both ICAM-1 and VCAM-1 were present in carotid arteries compared to control rats [21]. These 


\section{VCAM-1 Mediates Cigarette Smoke Extract Enhancement of Monocyte Adhesion to Human Carotid Endothelial Cells}

disparate effects of cigarette smoke on ICAM-1 and VCAM-1 expression profiles suggest that expression of these CAMs in response to cigarette smoke may be tissuespecific, and that vascular endothelial cells from one vascular compartment (e.g., peripheral pulmonary endothelium or HUVECs) will respond differently to smoke than other vascular compartments (e.g. carotid endothelium). In total, these reports and others suggest that CAM expression can be induced by smoking, and that this increased expression may be associated with the enhanced leukocyte-endothelial adhesion observed in early stages of atherosclerosis.

In this study, we investigated mechanisms by which CSE increased binding of monocytes to primary carotid vascular endothelial cells. We utilized CSE for these studies because this extract contains most of the particulates and chemical compounds found in cigarette smoke [22]. In order to determine the mechanisms by which smoking is involved with CVD and atherosclerosis, we investigated the effects of CSE on the expression of CAMs on human carotid endothelial cells (HCEC), cytokine production by HCEC, monocyte adhesion to HCEC, and migration of monocytes across HCEC monolayers.

In both time-dependent (Fig 1) and dose-response experiments (Fig 2), CSE significantly increased the surface expression of VCAM-1 in HCEC with slight increases in surface expression of ICAM-1. These results stand in contrast to other reports showing no increase in VCAM-1 expression in lung peripheral pulmonary endothelium or HUVECs [18], suggesting that carotid endothelium regulates CAM expression in response to CSE differently than other tissues. This further suggests that increased expression of VCAM-1, rather than ICAM-1, may play a more significant role in carotid plaque formation than in other vascular tissues.

We examined cytokine and chemokine production in HCEC exposed to CSE. Expression of cytokines and chemokines are important for recruitment of leukocytes to sites of eventual plaque formation. We were interested to determine if CSE would induce production of chemokines that could result in recruitment of key leukocyte mediators of atherosclerotic plaques. Using a $50 \mu \mathrm{g} / \mathrm{mL}$ dose of CSE at various times up to $48 \mathrm{hr}$ (Fig 3 ), we measured secreted levels of GMCSF, IL-6, IL-8, MCP-1, MCP-2, and RANTES. Interestingly, we found that compared to negative (no CSE) controls, CSE suppressed or had no effect on cytokine/chemokine production. Using a single time point of $24 \mathrm{hr}$ and various concentrations of CSE (Fig 4) we confirmed the results shown in Fig 3 demonstrating that CSE had no effect or suppressed cytokine production in HCEC. These data suggest that CSE plays little or no role on carotid endothelial recruitment of leukocytes through the induction of leukocytic chemotactic factors. Alternatively, the fact that CSE downregulates important pro-inflammatory responders may indicate that CSE decreases the capacity of the vasculature to quell inflammation, leading to the onset of atherosclerosis. Future studies will examine the effects of CSE on anti-inflammatory mediators such as IL-1 Receptor Antagonist (IL-1RA), IL-4, and IL-10.

We next examined whether CSE could increase adhesion of monocytes to an HCEC monolayer. Since CSE increased expression of surface VCAM-1, but not ICAM-1, in a dose-responsive manner (Fig 2 ), we predicted that monocyte adhesion would similarly increase in response to correspondingly higher concentrations of CSE. As shown in Fig 5, the number of monocytes that bound to HCEC increased in 


\section{VCAM-1 Mediates Cigarette Smoke Extract Enhancement of Monocyte Adhesion to Human Carotid Endothelial Cells}

the presence of CSE, up to 18 -fold above negative controls. Since even low concentrations $(5 \mu \mathrm{g} / \mathrm{mL})$ of CSE increased the numbers of bound monocytes, and this reflected the effects of similar CSE concentrations on VCAM-1, but not ICAM1, expression (Fig 2), these cumulative results suggest that VCAM-1, and not ICAM-1, is the key regulator of monocyte binding to HCEC in response to CSE exposure.

In follow-up experiments, we examined the ability of CSE to affect the number of monocytes that migrated across a monolayer of HCEC. We found that monocyte migration increased significantly with increasing concentrations of CSE (Fig 6). While this increase in transmigration capacity may simply reflect higher binding capacity of monocytes to endothelial surfaces due to increased expression of surface VCAM-1, we speculate that CSE may also influence monocyte migration function. We also cannot rule out that other endothelial surfaces molecules (such as
CD44, P-selectin, L-selectin, or E-selectin) that affect monocyte rolling and adhesion may also be affected by CSE. Future experiments will examine the effect of CSE on these molecules and monocytic activity.

More investigation is necessary to parse out the pathophysiology of atherosclerosis. This study reveals the interesting hypothesis that different vascular endothelial tissues respond differently to cigarette smoke, and drug therapies targeting CAM expression on endothelial cells may have to be specifically targeted to affected vasculature. Better understanding of the interactions between endothelial CAMs, cytokines, chemokines and monocytes will assist us in determining therapeutic targets that will allay this devastating disease.

\section{ACKNOWLEDGEMENTS}

This work was supported by the Research Service of the Department of Veterans Affairs (to M.L.G.), in which Dr. Goalstone is a recipient of a VA Merit Award. 
VCAM-1 Mediates Cigarette Smoke Extract Enhancement of Monocyte Adhesion to

\section{Human Carotid Endothelial Cells}

\section{FIGURE LEGENDS}

Fig 1

A.

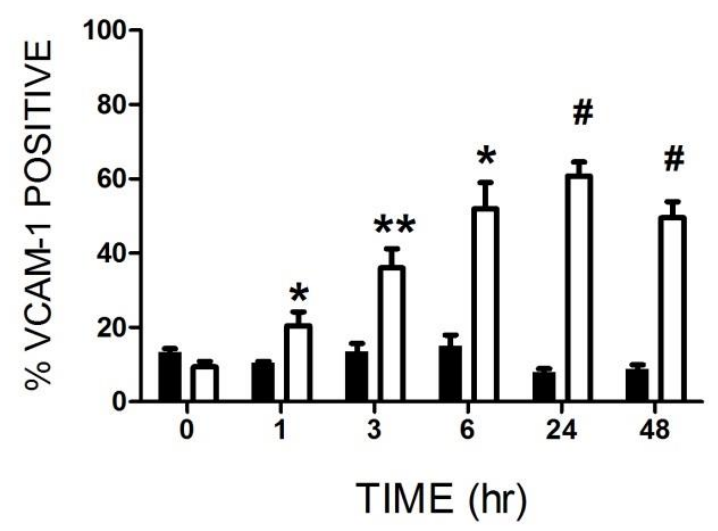

C.

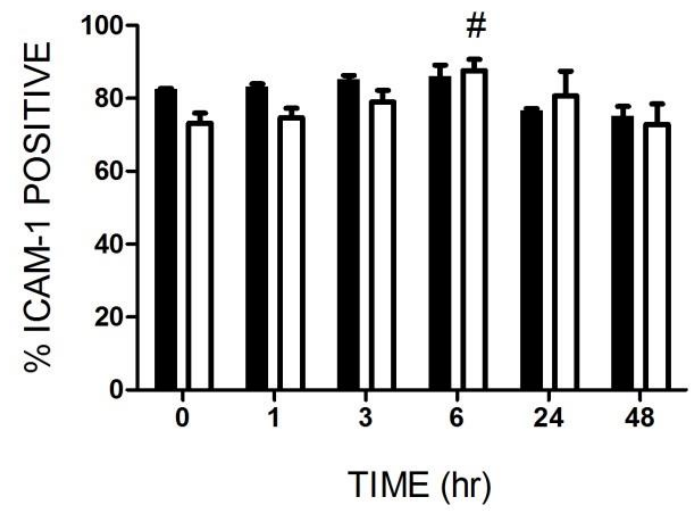

B.

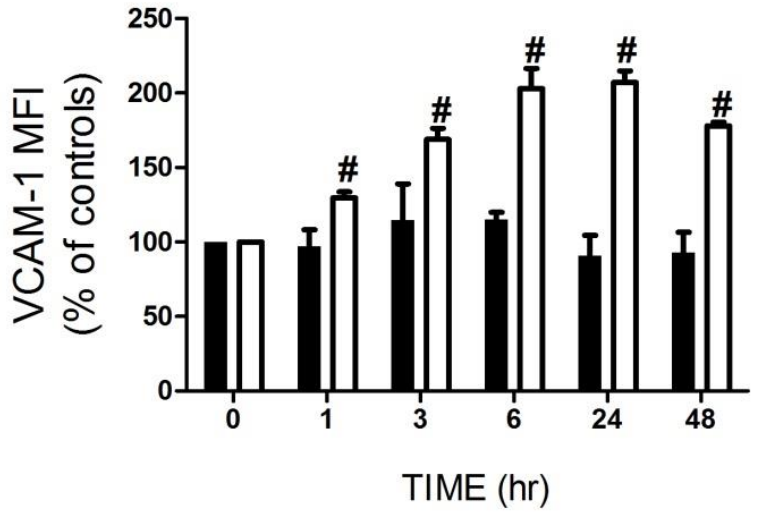

D.

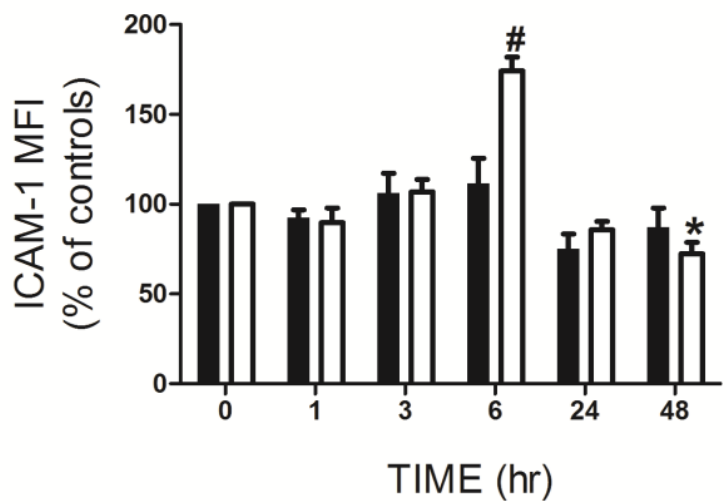

Figure \#1 -CSE increases the expression of VCAM-1 and ICAM-1 on HCEC in a timedependent manner. HCEC were incubated in medium in the absence or presence of CSE (50 $\mu \mathrm{g} / \mathrm{mL}$ ) for designated times. Cells were dissociated from the plates and suspensions were incubated with either PE-conjugated anti-VCAM-1 or APC-conjugated anti-ICAM-1 antibodies and subsequently analyzed by flow cytometry as noted in Methods. Black bars represent negative controls (cells not incubated in CSE) and open bars represent cells incubated in CSE. HCEC expressing CAM are expressed as either percent of total number of cells or mean fluorescent intensity (MFI). (A) Percent of HCEC that express VCAM-1 surface molecules. (B) Density (MFI) of VCAM-1 on HCEC. (C) Percent of cells that express ICAM-1 surface molecules. (D) Density (MFI) of ICAM-1 on HCEC. *, P < 0.05 vs zero time; **, $\mathrm{P}<0.01$ vs zero time; \#, $\mathrm{P}<0.005$ vs zero time; in four independent experiments. 
Fig 2

A.

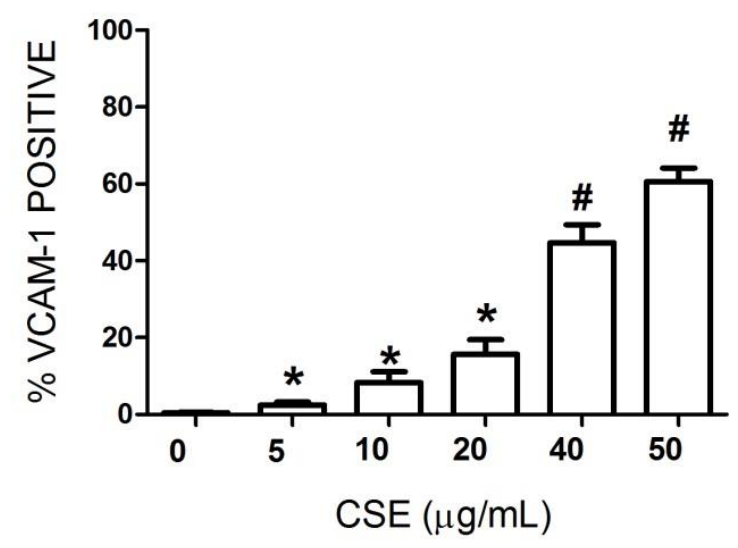

C.

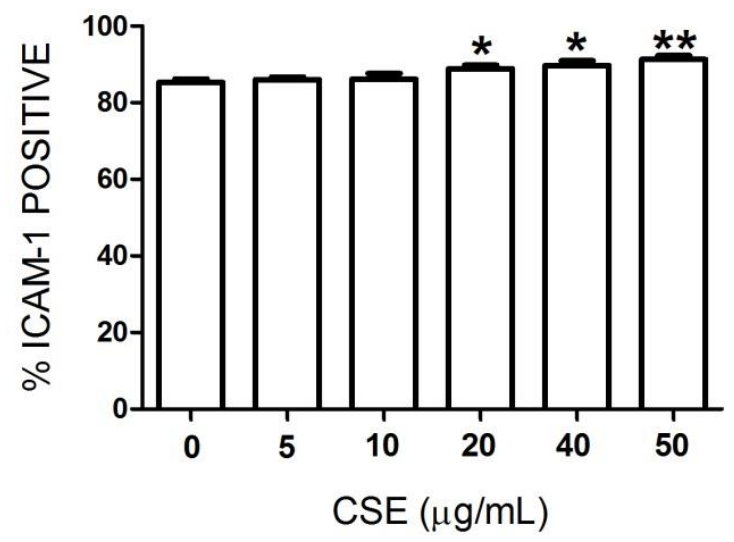

B.

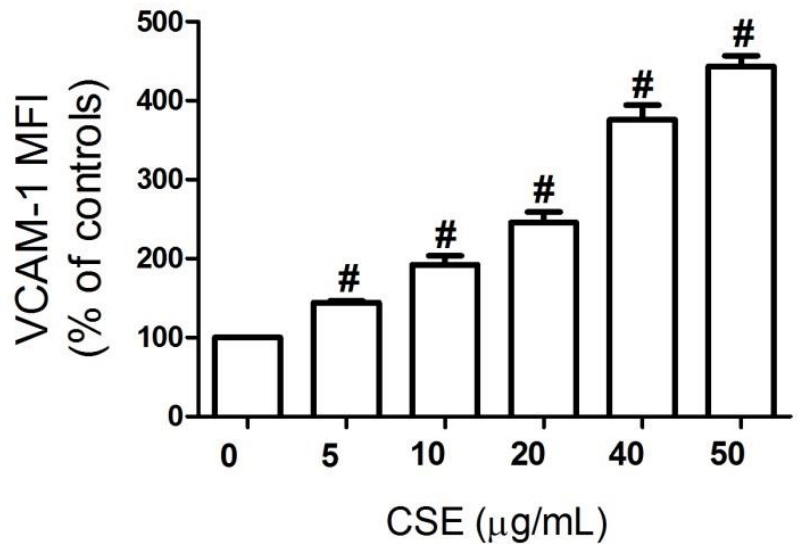

D.

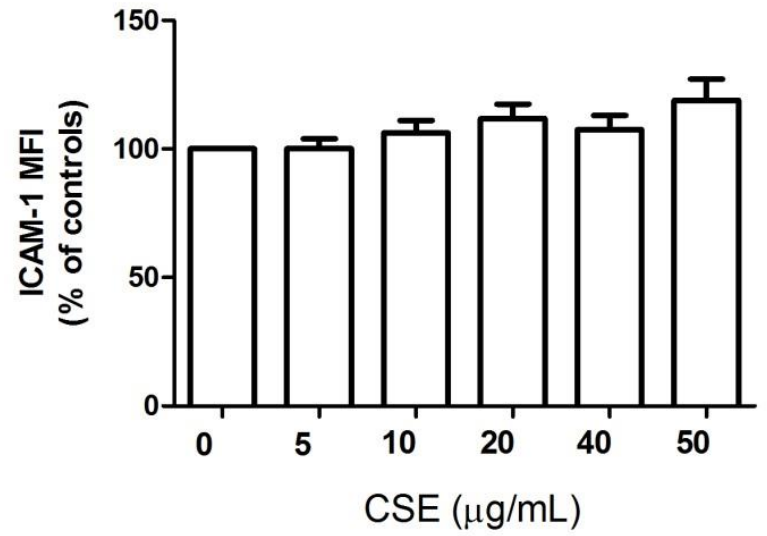

Figure \#2 - CSE induces expression of VCAM-1 and ICAM-1 on HCEC in a doseresponsive manner. HCEC were incubated $24 \mathrm{hr}$ in medium in the absence or presence of various concentrations of CSE. Cells were dissociated from the growth surface, exposed to fluorescent-conjugated anti-VCAM-1 or anti-ICAM-1 antibodies, and CAMs were measured by flow cytometry as noted in Methods. HCEC expressing CAM are expressed as either percent of total number of cells or MFI. (A) Percent of HCEC that express VCAM-1 surface molecules. (B) Density (MFI) of VCAM-1 on HCEC. (C) Percent of HCEC that express ICAM-1 surface molecules. (D) Density (MFI) of ICAM-1 on HCEC. *, $\mathrm{P}<0.05$ vs negative control (no CSE); **, $\mathrm{P}<0.01$ vs no CSE; \#, $\mathrm{P}<0.005$ vs no CSE; in five independent experiments. 
VCAM-1 Mediates Cigarette Smoke Extract Enhancement of Monocyte Adhesion to Human Carotid Endothelial Cells

Fig 3

A.

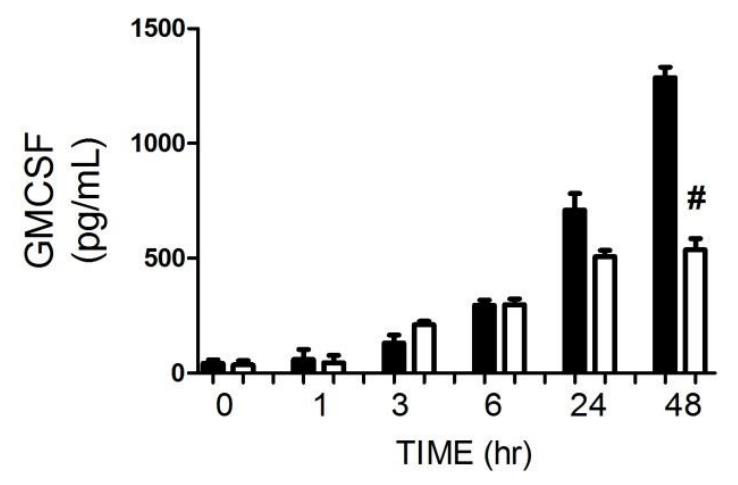

C.

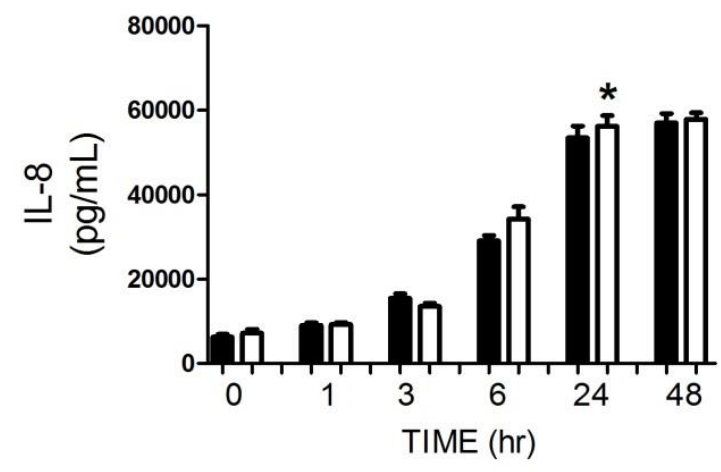

E.

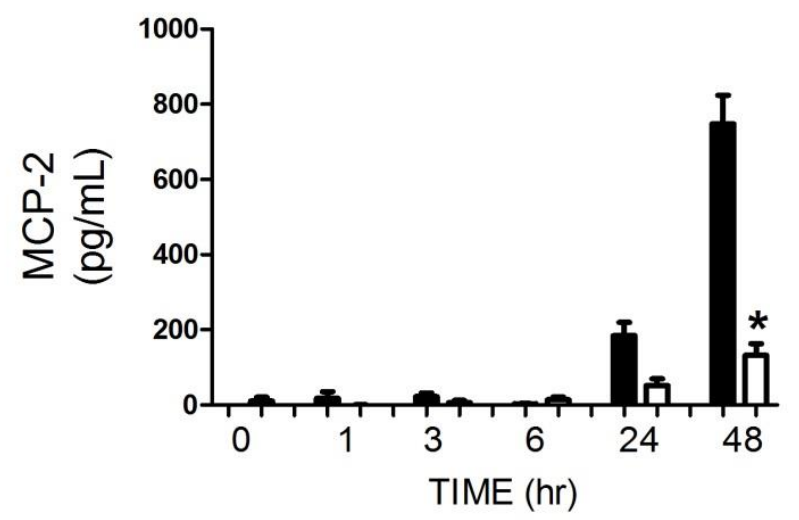

B.

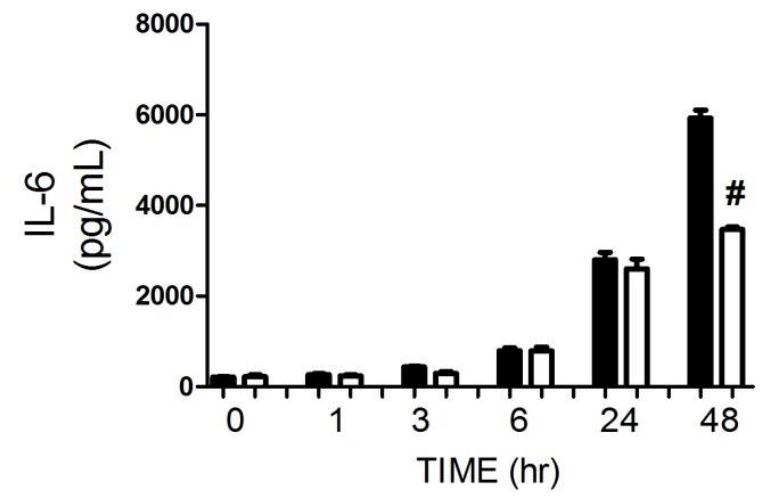

D.

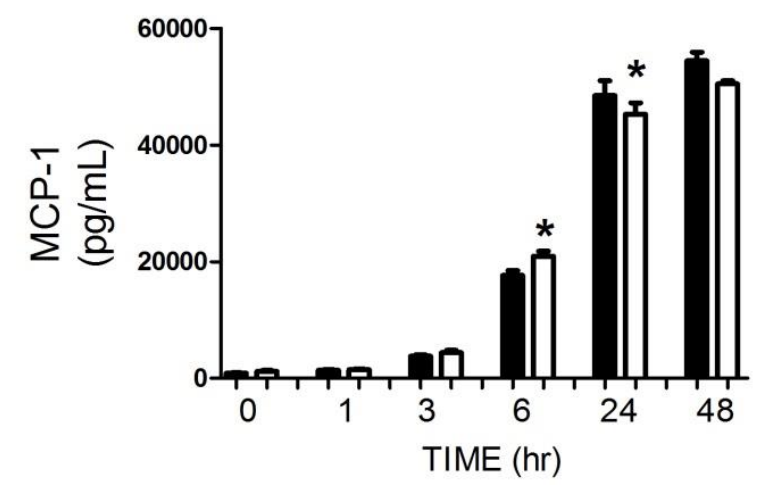

F.

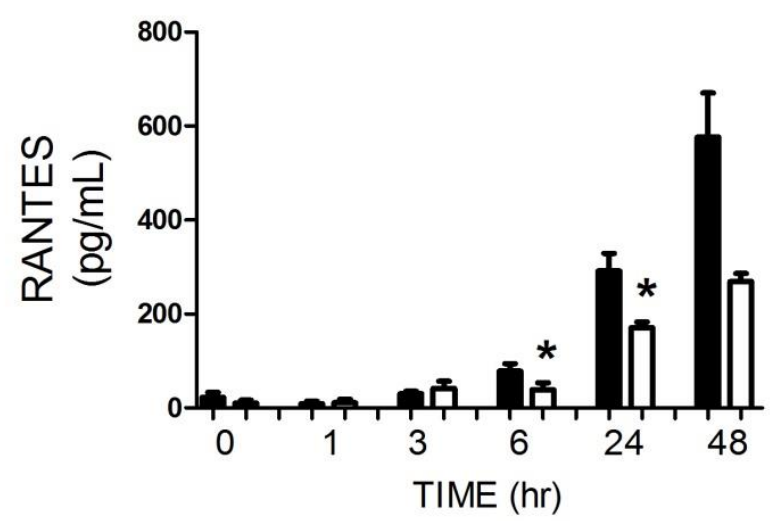

Figure \#3 - CSE suppresses expression of GMCSF, IL-6, IL-8, MCP-1, MCP-2, and RANTES in HCEC over time. HCEC were incubated in the absence (black bars) or presence (white bars) of CSE $(50 \mu \mathrm{g} / \mathrm{mL})$ for designated times. Cytokine expression was measured in cell-free supernatants using an ELISA assay as described in Methods. *, $\mathrm{P}<0.05$ vs negative (no CSE) controls at the same time point; \#, P $<0.005$ vs negative controls (no CSE) at the same time point. Concentrations of cytokines are expressed in $\mathrm{pg} / \mathrm{mL}$ and represent the mean $\pm \mathrm{SEM}$ of three independent experiments. 
VCAM-1 Mediates Cigarette Smoke Extract Enhancement of Monocyte Adhesion to Human Carotid Endothelial Cells

Fig 4

A.

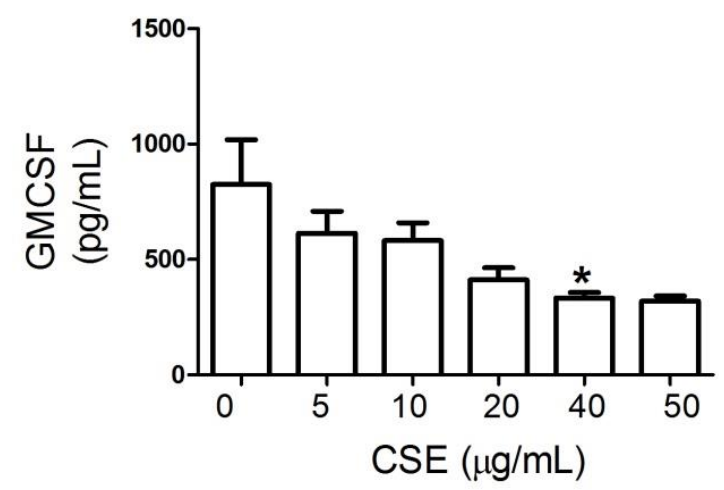

C.

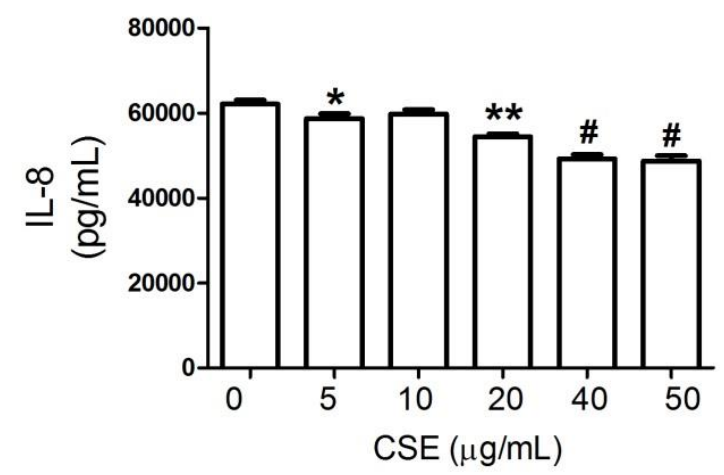

E.

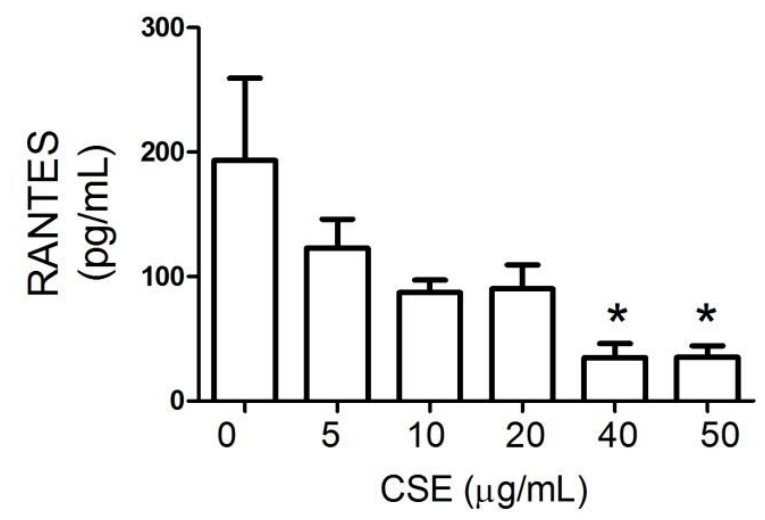

B.

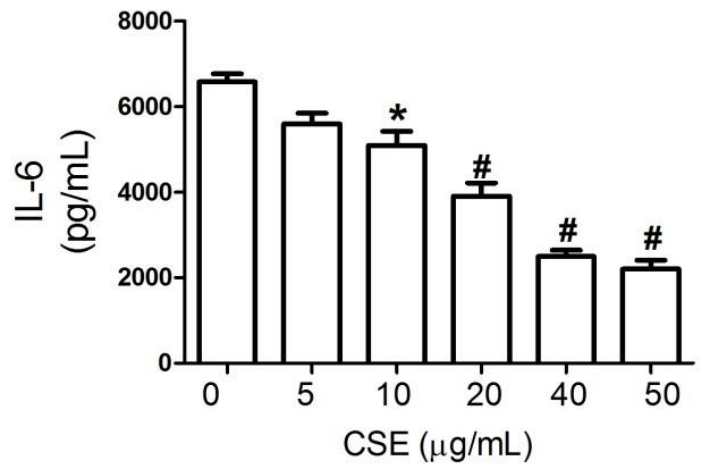

D.

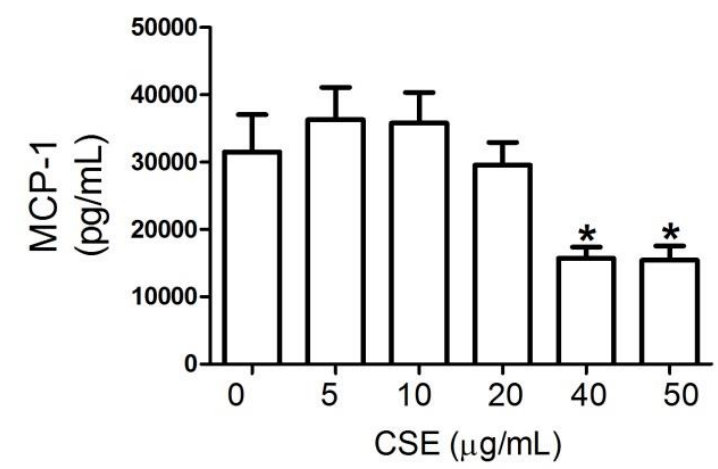

Figure \#4 - Increased CSE concentrations decrease expression of cytokines and chemokines expressed by HCEC. HCEC were challenged with CSE $(0-50 \mu \mathrm{g} / \mathrm{mL})$ for $24 \mathrm{hr}$. Cytokine expression was measured in cell-free supernatants using an ELISA assay as described in Methods. *, $\mathrm{P}<0.05$ vs negative controls (no CSE); \#, $\mathrm{P}<0.005$ vs no CSE. Concentrations of cytokines are expressed in $\mathrm{pg} / \mathrm{mL}$ and represent the mean $\pm \mathrm{SEM}$ of five independent experiments. 
VCAM-1 Mediates Cigarette Smoke Extract Enhancement of Monocyte Adhesion to Human Carotid Endothelial Cells

Fig 5

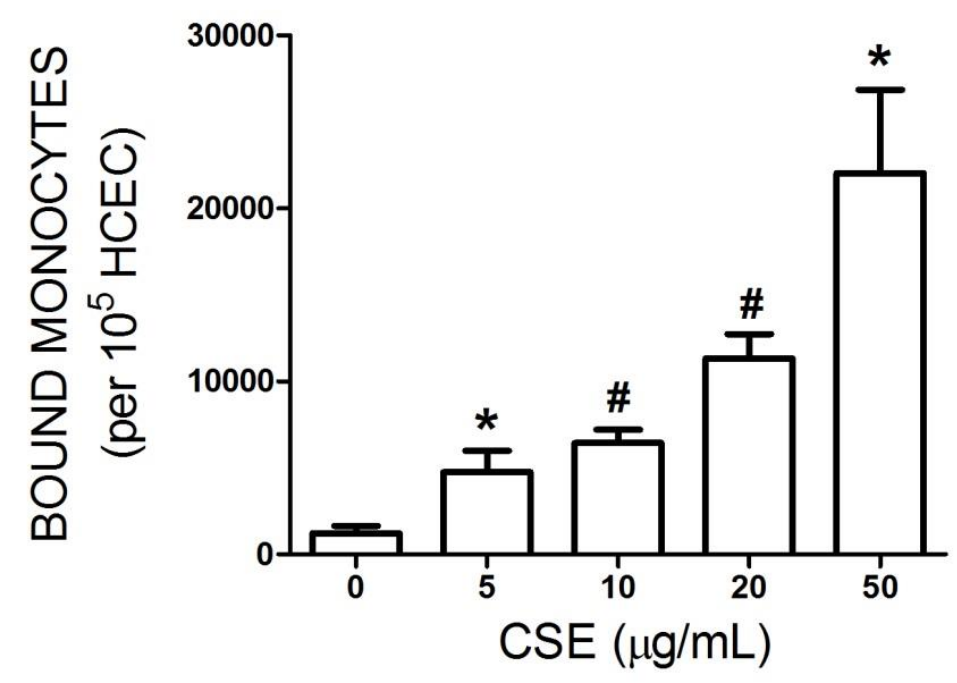

Figure \#5 - CSE increases the number of monocytes that bind to HCEC. CSE $(0-50$ $\mu \mathrm{g} / \mathrm{mL}$ ) was introduced to confluent HCEC for $24 \mathrm{~h}$. After washing HCEC twice with PBS, $1 \mathrm{x}$ $10^{6}$ U937 cells were added to the medium and the cells were allowed to adhere to HCEC. Unbound U937 cells were removed and conjugated U937/HCEC were suspended and U937 cells were stained with FITC-conjugated anti-human CD14 antibody. The numbers of bound monocytes were determined by flow cytometry and expressed as number of monocytes per $1 \mathrm{x}$ $10^{5}$ HCEC (unstained cells). The graph represents the mean \pm SEM of five independent experiments. *, $\mathrm{P}<0.05$ vs negative controls (no CSE); \#, $\mathrm{P}<0.005$ vs negative controls (no CSE). 
VCAM-1 Mediates Cigarette Smoke Extract Enhancement of Monocyte Adhesion to Human Carotid Endothelial Cells

Fig 6

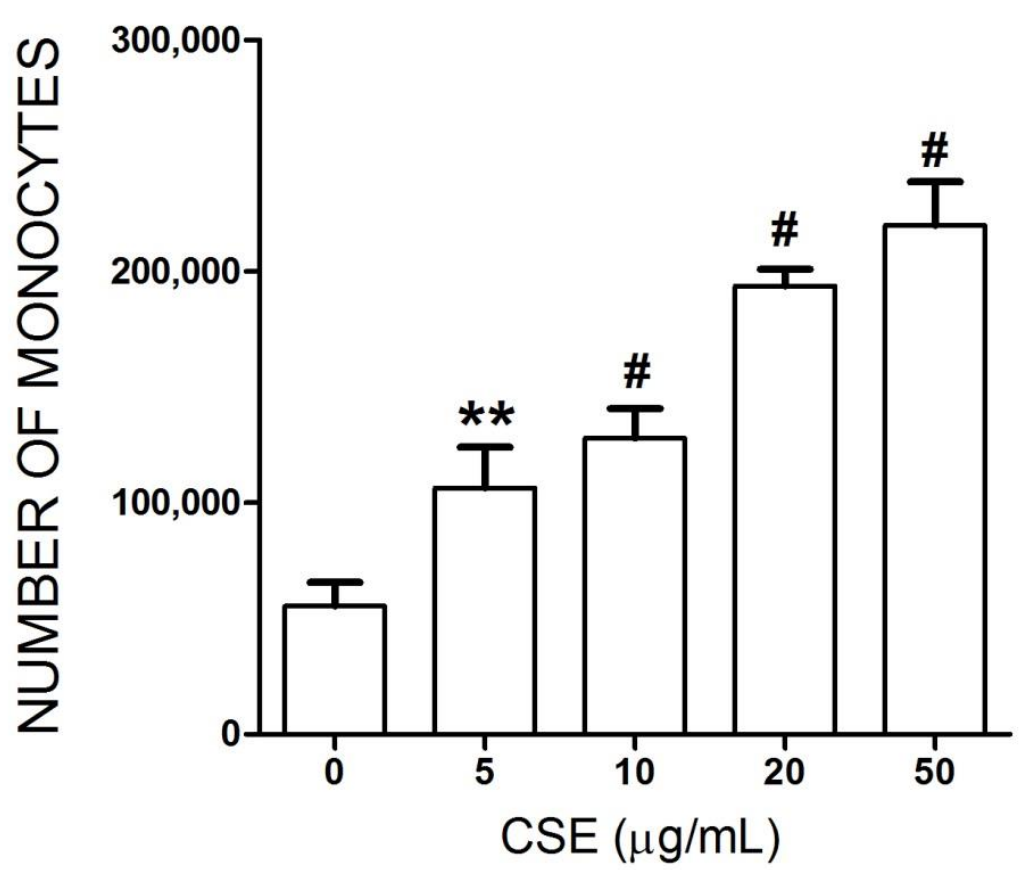

Figure \# 6 - CSE increases the number of U937 monocytes that transmigrate through a monolayer of HCEC. HCEC were cultured in MGM on $8 \mu \mathrm{m}$ pore size membrane inserts until confluent. MCP-1 was added to the bottom chamber and CSE $(0-50 \mu \mathrm{g} / \mathrm{mL})$ was added to the top chamber. After adding $1 \times 10^{6}$ of U937cells to the top chamber, the co-cultures were incubated for $48 \mathrm{hr}$. The migrated U937 cells present in the bottom chamber were centrifuged and stained with FITC-conjugated anti-human CD14 antibody, and the total number of FITCstained U937 cells was quantified using flow cytometry. Graphs are expressed in number of transmigrated monocytes and represent the mean \pm SEM for four experiments. **, $\mathrm{P}<0.01$ vs negative controls (no CSE); \#, P 0.005 vs negative controls (no CSE). 


\section{VCAM-1 Mediates Cigarette Smoke Extract Enhancement of Monocyte Adhesion to Human Carotid Endothelial Cells}

\section{REFERENCES}

1. Hsueh WA and Law RE (1999) Insulin signaling in the arterial wall. Am J Cardiol 84: 21J-24J.

2. Prescott SM, McIntyre TM and Zimmerman GA (2001) Events at the vascular wall: The molecular basis of inflammation. J. Invest. Med. 49: 104111.

3. Ley K, Miller YI and Hedrick CC (2011) Monocyte and macrophage dynamics during atherogenesis. Arterioscler Thromb Vasc Biol 31: 1506-1516.

4. Breen DM, Chan KK, Dhaliwall JK, Ward MR, Al Koudsi N, Lam L, De Souza M, Ghanim H, Dandona P, Stewart DJ, Bendeck MP and Giacca A (2009) Insulin increases reendothelialization and inhibits cell migration and neointimal growth after arterial injury. Arterioscler Thromb Vasc Biol 29: 1060-1066.

5. Standl E (1995) Hyperinsulinemia and atherosclerosis. Clin Invest Med 18: 261-266.

6. Endemann DH and Schiffrin EL (2004) Endothelial dysfunction. J Am Soc Nephrol 15: 1983-1992.

7. Nana-Sinkam SP, Lee JD, SottoSantiago S, Stearman RS, Keith RL, Choudhury Q, Cool C, Parr J, Moore MD, Bull TM, Voelkel NF and Geraci MW (2007) Prostacyclin prevents pulmonary endothelial cell apoptosis induced by cigarette smoke. Am J Respir Crit Care Med 175: 676-685.

8. Horvathova M, Jahnova E, Szabova M, Tulinska M, Kuricova M, Liskova
A, Volkovova $\mathrm{K}$ and Dusinska $\mathrm{M}$ (2009) The relationship between cell surface markers, cytokines, ageing, and cigarette smoking. Bratisl Lek Listy 110: 394-400.

9. Cybulsky MI, Iiyama K, Li H, Zhu S, Chen M, Iiyama $M$, Davis $V$, Gutierrez-Ramos JC, Connelly PW and Milstone DS (2001) A major role for VCAM-1, but not ICAM-1, in early atherosclerosis. J Clin Invest 107: 1255-1262.

10. Singh RB, Mengi SA, Xu YJ, Arneja AS and Dhalla NS (2002) Pathogenesis of atherosclerosis: A multifactorial process. Exp Clin Cardiol 7: 40-53.

11. Madonna $\mathrm{R}$, Massaro $\mathrm{M}$ and De Caterina R (2008) Insulin potentiates cytokine-induced VCAM-1 expression in human endothelial cells. Biochim Biophys Acta 1782: 511-516.

12. Scott DA and Palmer RM (2002) The influence of tobacco smoking on adhesion molecule profiles. Tob Induc Dis 1:7-25.

13. Selya AS and Hesse ND (2017) Time to first cigarette and serum cholesterol levels. Soc Sci Med 174: 213-219.

14. Maranhao RC and Leite AC (2014) Development of Anti-Atherosclerosis Therapy Based on the Inflammatory and Proliferative Aspects of the Disease. Curr Pharm Des

15. Selvin E, Halushka MK, Rawlings AM, Hoogeveen RC, Ballantyne CM, Coresh J and Astor BC (2013) sRAGE and risk of diabetes, cardiovascular 


\section{VCAM-1 Mediates Cigarette Smoke Extract Enhancement of Monocyte Adhesion to Human Carotid Endothelial Cells}

disease, and death. Diabetes 62: 21162121.

16. Zhu T, Zhang L, Ling S, Duan J, Qian $\mathrm{F}, \mathrm{Li} \mathrm{Y}$ and $\mathrm{Xu}$ JW (2014) Scropolioside B Inhibits IL-1 beta and Cytokines Expression through NFkappa B and Inflammasome NLRP3 Pathways. Mediators Inflamm 2014: 819053.

17. Erusan RR, Nalini D, Ganesan M and Malathi R (2012) Correlation between Obesity and Inflammation in Cardiovascular Diseases - Evaluation of Leptin and Inflammatory Cytokines. Open Journal of Endocrine and Metabolic Diseases 2: 7-15.

18. Schaberg T, Rau M, Oerter R, Liebers U, Rahn W, Kaiser D, Witt C and Lode H (1996) Expression of adhesion molecules in peripheral pulmonary vessels from smokers and nonsmokers. Lung 174: 71-81.

19. Adams MR, Jessup W and Celermajer DS (1997) Cigarette smoking is associated with increased human monocyte adhesion to endothelial cells: reversibility with oral L-arginine but not vitamin C. J Am Coll Cardiol 29: 491-497.

20. Shen Y, Rattan V, Sultana C and Kalra VK (1996) Cigarette smoke condensate-induced adhesion molecule expression and transendothelial migration of monocytes. Am J Physiol 270: H16241633.

21. Yang GH, Li YC, Wang ZQ, Liu B, Ye W, Ni L, Zeng R, Miao SY, Wang LF and Liu CW (2014) Protective effect of melatonin on cigarette smoke-induced restenosis in rat carotid arteries after balloon injury. J Pineal Res 57: 451-458.

22. Rao P, Ande A, Sinha N, Kumar A and Kumar S (2016) Effects of Cigarette Smoke Condensate on Oxidative Stress, Apoptotic Cell Death, and HIV Replication in Human Monocytic Cells. PLoS One 11: e0155791. 